\title{
DRIVING CYCLES SELF-RECOGNITION OF HEV BASED ON MULTI-AGENT THEORY
}

\author{
Limin Niu' ${ }^{1}$ Lijun Ye ${ }^{2}$ and Hongyuan Yang ${ }^{3}$ \\ ${ }^{1,2,3}$ School of Mechanical Engineering \\ Anhui University of Technology \\ 243032 Maanshan, China \\ Email: niulimin@ahut.edu.cn; ylj860119@163.com; d133566410@163.com \\ DOI: https://doi.org/10.22452/mjcs.vol32no3.5
}

\begin{abstract}
In wiew of the current control strategies of Hybrid Electric Vehicle (HEV) that cannot satisfies the requirement of complex driving cycles, a driving cycles self-recognition strategy based on multi-agent theory is presented. Driving cycles recognition agent, powertrain agent, and system agent are built to form a multi-agent system. Based on the mean vehicle speed, absolute mean acceleration and standard variance of acceleration, current driving cycles are recognized by fuzzy logic controller. Powertrain adaptive matching control for current driving cycles is realized by coordination with the system agent. Simulation model is established in Advisor software. Driving cycle of the New European Driving Cycle (NEDC) and CHINA are chosen as the experiment driving cycles. The results show that driving cycles self-recognition strategy of HEV based on multi-agent theory can recognize current driving cycle accurately. Powertrain coordination matching control is realized. Vehicle performance and intellectualization are improved further.
\end{abstract}

Keywords: Hybrid electric vehicle, Multi-agent system, Driving cycles self-recognition, Fuzzy logic

\subsection{INTRODUCTION}

Hybrid Electric Vehicle (HEV) is more and more valued by auto industry because of the advantage in reducing fuel consumption and emissions[1,2]. Reasonable power train control strategy development is the core of realization of energy conservation and emissions reduction by HEV[3,4]. There are many control strategies applied to HEV in recent years, such as: trajectory optimization control strategy[5], best engine fuel consumption point control strategy[6], hierarchical control strategy based on CANopen protocol[7], multi-objective optimization fuzzy control strategy based on genetic algorithm[8], and so on. These control strategies overlook the influence of the driving cycles on vehicle performance and cannot adapt to complex and various driving cycles, as the current control strategies lack of the necessary intelligence. Driving cycles self-recognition of Parallel Hybrid Electric Vehicle (PHEV) based on the multi-agent theory is presented in order to improve the driving cycles self-recognition and intelligence of the control strategy.

The remainder of the paper is organized as follows. Section 2 describes the multi-agent coordination control system of HEV. In section 3, driving cycles recognition was investigated, which included driving intention fuzzy recognition and driving cycles fuzzy recognition. Simulation analysis was carried out in section 4 . Finally, section 5 concludes the paper.

\subsection{MULTI-AGENT COORDINATION CONTROL SYSTEM}

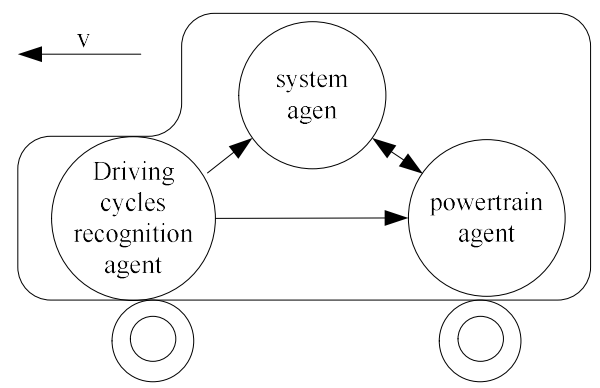

Fig. 1: Multi-agent coordination control system 
Fig. 1 shows the multi-agent coordination control system of HEV based on multi-agent theory, where it consists of driving cycles recognition agent, system agent, and powertrain agent. Driving cycles recognition agent is responsible for the driving cycles self-recognition. System agent is responsible for the overall coordination, and power train agent is responsible for the power matching. The agents transmit information to each other and work cooperatively in order to improve vehicle performance. This paper only describes the driving cycles recognition agent due to limited space.

\subsection{DRIVING CYCLES RECOGNITION}

Driving cycles are divided into rural driving cycle (Rdc), urban driving cycle (Udc), and expressway driving cycle (Edc). Fig. 2 shows the driving cycles recognition agent model, and the principle of driving cycles recognition can be described as follows. Driving cycles recognition agent perceives the current vehicle speed and calculates the mean speed, mean acceleration and acceleration standard deviation. These parameters are regarded as the inputs of a fuzzy logic controller to recognize the driving intention, which is divided into dynamic property and economy[9]. Then, the driving cycles are recognized and the information of driving cycles is transmitted to the agent system and power train agent. The reasoning process is described as follows.

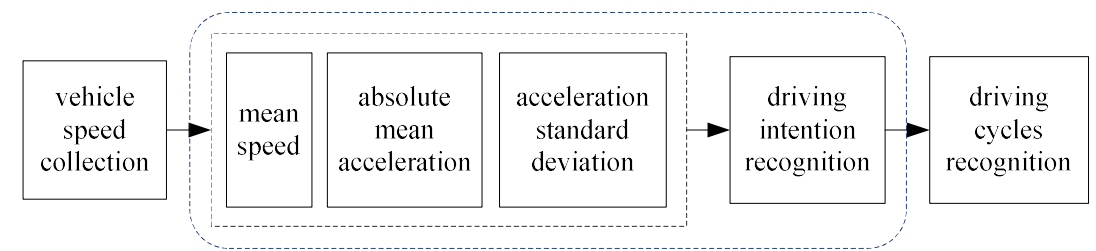

Fig.2: Driving cycles recognition agent model

\subsection{Driving Intention Fuzzy Recognition}

Acceleration average value (Aav), acceleration standard deviation (Asd) are calculated based on the vehicle speed. Driving intention is identified by a fuzzy logic controller which puts Aav and Asd as inputs and driving model (MS) as output (driving model means driving intention). Driving intention fuzzy recognition model is shown as Fig. 3.

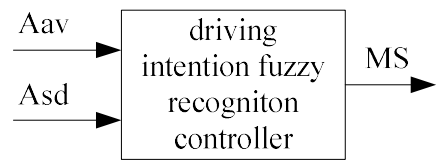

Fig. 3: Driving intention fuzzy recognition model

NEDC driving cycle, which is similar to urban and expressway driving cycles in China. CHINA driving cycle is mainly urban driving cycle in this work, and it is chosen to determine the fuzzy sets domain of Aav. The main parameters such as mean speed, maximum speed, mean acceleration, maximum acceleration, and so on are different as shown in Table 1.

Table 1: The parameters of NEDC and CHINA

\begin{tabular}{|l|c|l|c|}
\hline \multicolumn{2}{|c|}{ NEDC driving cycle } & \multicolumn{2}{c|}{ CHINA driving cycle } \\
\hline Parameters & Value & Parameters & Value \\
\hline simulation time $/(\mathrm{s})$ & 1184 & simulation time $/(\mathrm{s})$ & 1313 \\
\hline distance $/(\mathrm{km})$ & 10.93 & distance $/(\mathrm{km})$ & 60 \\
\hline max speed $/(\mathrm{km} / \mathrm{h})$ & 120 & max speed $/(\mathrm{km} / \mathrm{h})$ & 16.16 \\
\hline mean speed $/(\mathrm{km} / \mathrm{h})$ & 33.21 & mean speed $/(\mathrm{km} / \mathrm{h})$ & 0.91 \\
\hline max acceleration $/\left(\mathrm{m} / \mathrm{s}^{2}\right)$ & 1.06 & max acceleration $/\left(\mathrm{m} / \mathrm{s}^{2}\right)$ & 0.3 \\
\hline mean acceleration $/\left(\mathrm{m} / \mathrm{s}^{2}\right)$ & 0.54 & mean acceleration $/\left(\mathrm{m} / \mathrm{s}^{2}\right)$ & 381 \\
\hline idle time $/(\mathrm{s})$ & 298 & idle time $/(\mathrm{s})$ & 14 \\
\hline stop frequency & 13 & stop frequency & \\
\hline
\end{tabular}


Fig. 4 and Fig. 5 show the required real-time speed (v) and absolute Aav of NEDC and CHINA driving cycles. As shown Fig. 4 and Fig. 5, Aav of the two driving cycles are mostly in the interval [0,1]. The statistical points of Aav $>1$ can be regarded as the statistical points of $A a v=1$, which are points of big mean acceleration. So, fuzzy sets domain of Aav is defined as [0,1], and fuzzy language variables sets are defined as $\{\mathrm{S}, \mathrm{Z}, \mathrm{B}\}$, that is $\{\mathrm{Small}$, Medium, Big\}. Membership function of fuzzy language variables sets of Aav in domain of discourse is shown in Fig. 6. To avoid inappropriate repetition, it adopted a triangular model as membership function of sets of S, Z, and B.
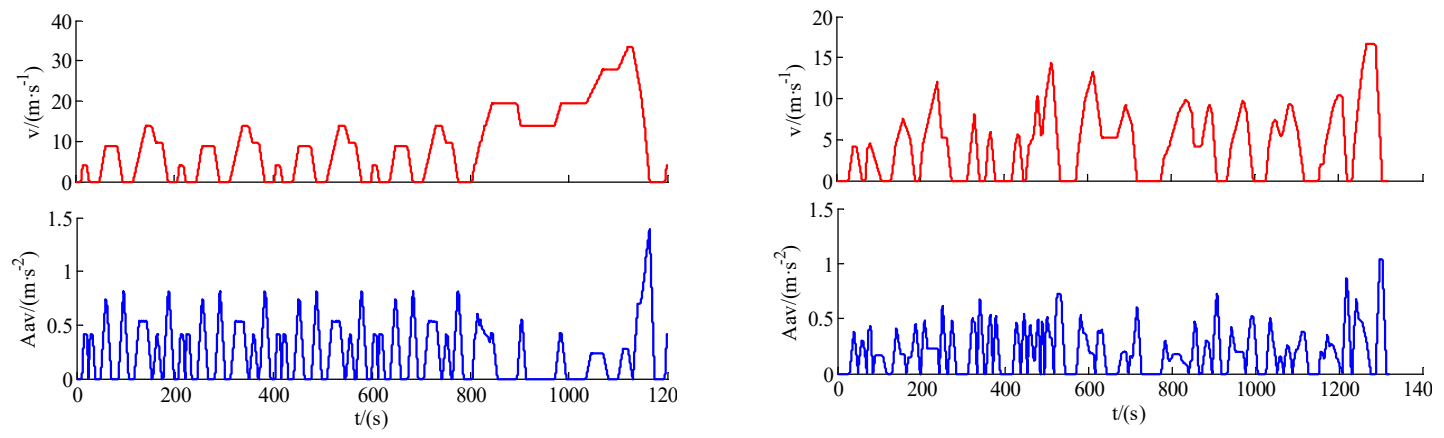

Fig. 4: NEDC driving cycle

Fig. 5: CHINA driving cycle

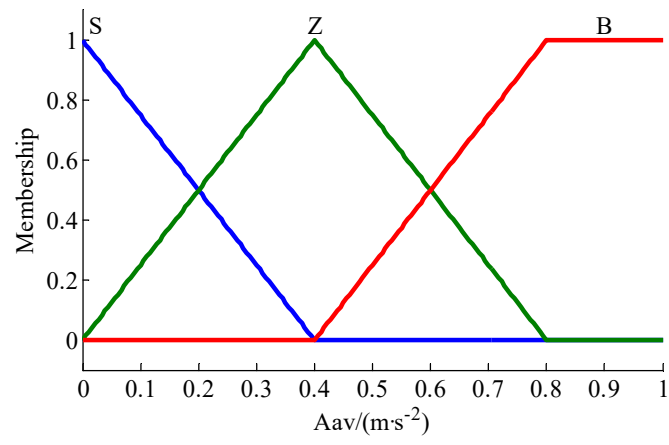

Fig. 6: Membership function of Aav

Asd is converted into the interval $[0,1]$ using normalization, so, universe of fuzzy sets of Asd is defined as [0,1], and fuzzy language variables sets are defined as $\{\mathrm{S}, \mathrm{Z}, \mathrm{B}\}$, that is $\{$ Small, Medium, Big $\}$. Membership function of fuzzy language variables sets of Asd in domain of discourse is shown as Fig. 7. To avoid inappropriate repetition, it adopted triangular model as membership function of sets of S, Z, and B, as shown in Fig. 6. Universe of fuzzy sets of MS is defined as $[0,1]$, and fuzzy language variables sets are defined as $\{E E, P P\}$, that is $\{$ Economical-Efficiency, Power-Performance\}. Membership function of fuzzy language variables sets of MS in domain of discourse is shown in Fig. 8. Economical-Efficiency or Power-Performance is only used for the simple judgment for MS, so trapezoidal model membership function was adopted for its simple form.

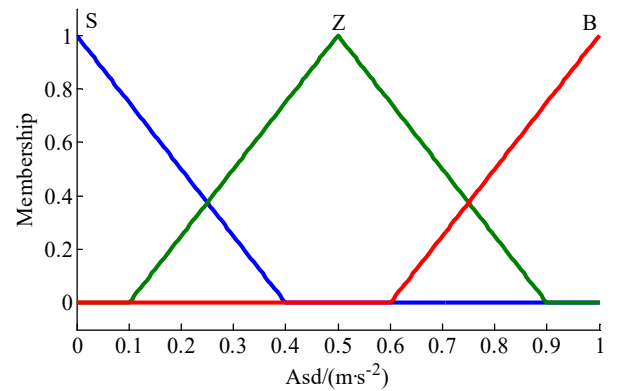

Fig. 7: Membership function of Asd

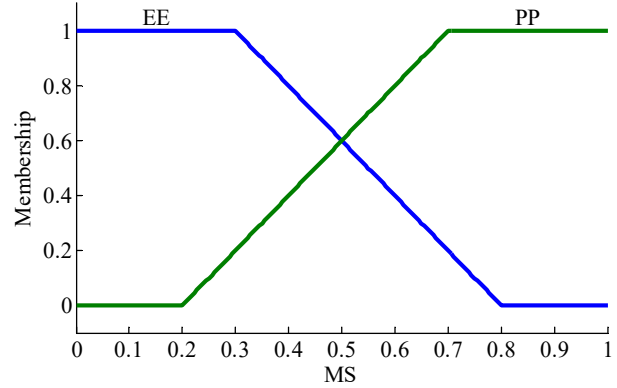

Fig. 8: Membership function of driving model

MS can be recognized through a fuzzy logic controller which regards Aav and Asd as inputs, and fuzzy logic rules are shown as Table 2. Fuzzy logic rules can be described as follows: IF (Aav is S) and (Asd is S), THEN (MS is $\mathrm{EE})$, that is if acceleration average value is small, and acceleration standard deviation is small, then it means current driving model is inclined to economical-efficiency....IF (Aav is B) and (Asd is Z), THEN (MS is PP).

Table 2: Fuzzy logic rules of driving model 


\begin{tabular}{|c|c|c|c|c|}
\hline \multicolumn{2}{|c|}{} & \multicolumn{3}{c|}{ Aav } \\
\cline { 3 - 5 } \multicolumn{2}{|c|}{} & S & Z & B \\
\hline \multirow{3}{*}{ Asd } & S & EE & EE & PP \\
\cline { 2 - 5 } & Z & EE & EE & PP \\
\cline { 2 - 5 } & B & EE & PP & PP \\
\hline
\end{tabular}

\subsection{Driving Cycles Fuzzy Recognition}

Driving cycles (DC) can be recognized through a fuzzy logic controller that regards MS and speed average value (Sav) as inputs after obtaining the driving model, which places emphasis on economical efficiency or power performance. Driving cycles fuzzy recognition model is shown in Fig. 9.

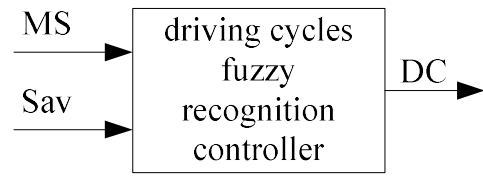

Fig. 9: Driving cycles fuzzy recognition model

The highest design speed in this paper is $160 \mathrm{~km} / \mathrm{h}$, about $45 \mathrm{~m} / \mathrm{s}$. Thus universe of fuzzy sets of Sav is defined as [0,45] (unit is $\mathrm{m} / \mathrm{s}$ ), and fuzzy language variables sets are defined as $\{$ LS MS HS\}, that is \{Low-speed Mediumspeed High-speed \}. Membership function of fuzzy language variables sets of Sav in domain of discourse is shown as Fig. 10, membership function of MS is triangular model, and membership functions of LS and HS are trapezoidal model.

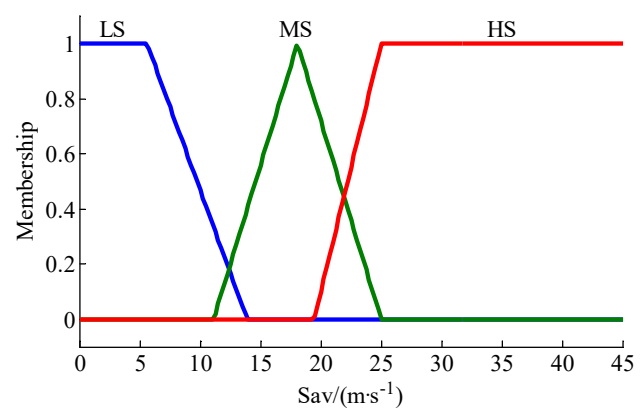

Fig. 10: Membership function of Sav

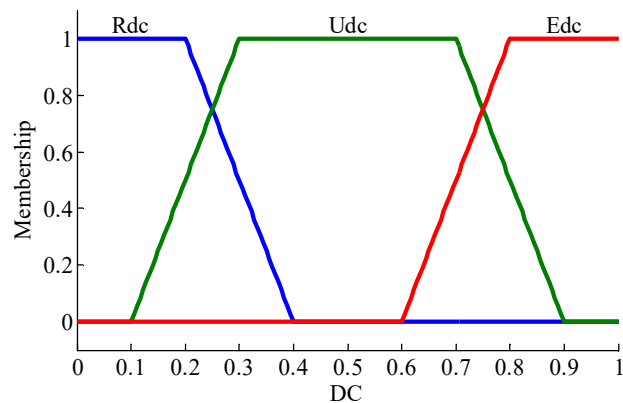

Fig. 11: Membership function of driving cycles

Universe of fuzzy sets of DC is defined as [0,1], and fuzzy language variables sets are defined as mentioned above $\{$ Rdc Udc Edc $\}$. Membership function of fuzzy language variables sets of DC in domain of discourse is shown in Fig. 11. For simplicity, trapezoidal model is adopted for membership function of driving cycles. Fuzzy logic rules of driving cycles is shown as Table 3, the rules can be described as follows: IF (MS is EE) and (Sav is LS), THEN (DC is $\mathrm{Udc}$ ), that is if driving model is inclined to economical-efficiency, and speed average value is low, then means driving cycles perharps is urban driving cycle.... IF (MS is PP) and (Sav is MS), THEN (DC is Rdc)...

Table 3: Fuzzy logic rules of driving cycles

\begin{tabular}{|c|c|c|c|}
\hline \multicolumn{2}{|c|}{-} & \multicolumn{2}{c|}{ MS } \\
\cline { 3 - 4 } Sav & LS & EE & PP \\
\cline { 2 - 4 } & MS & Udc & Rdc \\
\cline { 2 - 4 } & HS & Edc & Edc \\
\hline
\end{tabular}

Driving cycles are recognized through a fuzzy logic controller, which regards Sav and MS as inputs. Driving cycles recognized are some defuzzification numbers in universe of fuzzy sets of DC. The logic threshold rules are formulated depending on the membership of $\mathrm{DC}$, which can be described as follows: If $0 \leqslant \mathrm{DC}<0.25$, then driving cycle is rural driving cycle and marked as 1 ; If $0.25 \leqslant \mathrm{DC}<0.75$, then driving cycle is urban driving cycle and 
marked as 2 ; If $0.75 \leqslant \mathrm{DC} \leqslant 1$, then driving cycle is expressway driving cycle and marked as 3 . Table 4 shows the identity value of driving cycles.

Table 4: The defined table of driving cycles

\begin{tabular}{|c|c|c|}
\hline Driving cycles & Defuzzification range & Identity value \\
\hline $\mathrm{Rdc}$ & {$[0,0.25)$} & 1 \\
\hline $\mathrm{Udc}$ & {$[0.25,0.75)$} & 2 \\
\hline $\mathrm{Edc}$ & {$[0.75,1]$} & 3 \\
\hline
\end{tabular}

\subsection{SIMULATION AND ANALYSIS}

To verify the validity of the driving cycles recognition agent, driving cycles recognition agent model is established using MATLAB/Simulink. Vehicle performance evaluation is conducted using the formulated driving cycles fuzzy recognition rules and driving cycles recognition simulation.

\subsection{Driving Cycles Self-recognition Simulation}

Fig. 12 shows the driving cycles self-recognition model based on the multi-agent theory. It is composed of several sub-modules, such as mean speed, mean acceleration, acceleration standard deviation, driving intention fuzzy recognition, and driving cycles fuzzy recognition. Recognition results of NEDC and CHINA are shown in Fig. 13 and Fig. 14 respectively, followed with the analysis of the results.

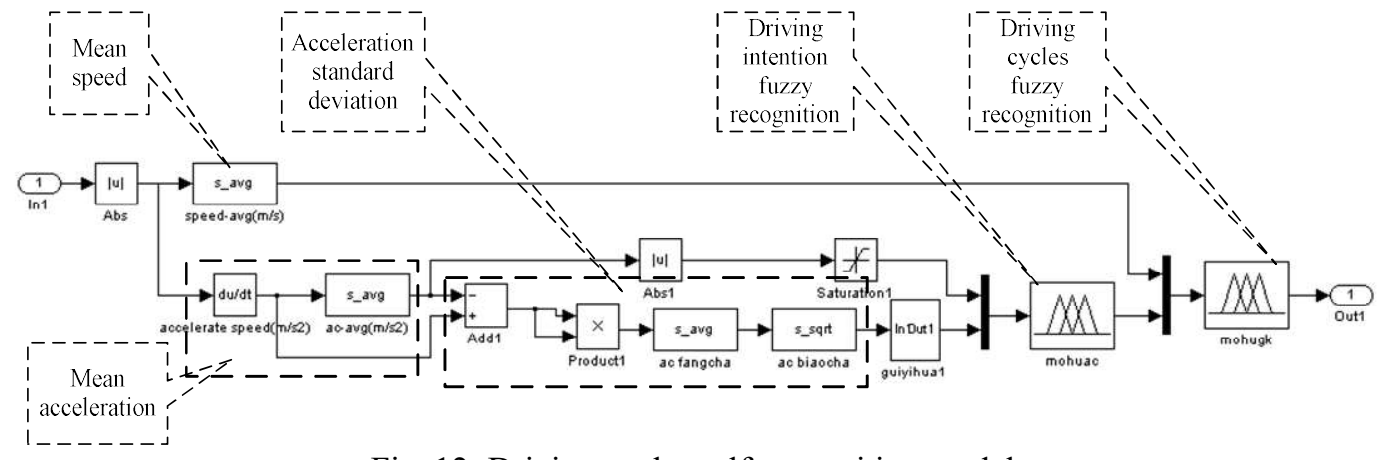

Fig. 12: Driving cycles self-recognition model
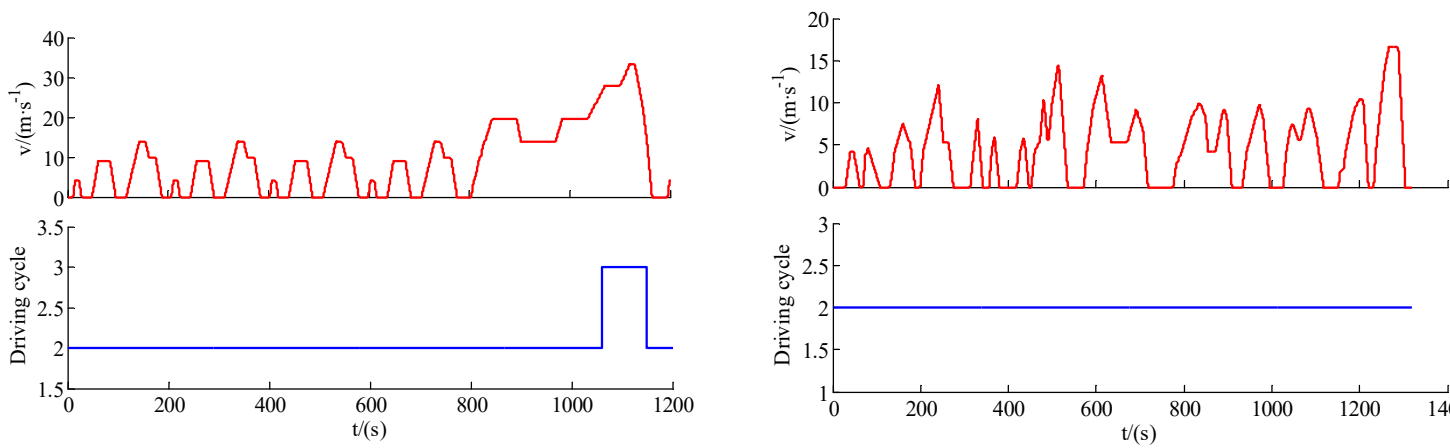

Fig. 13: Recognition result of NEDC

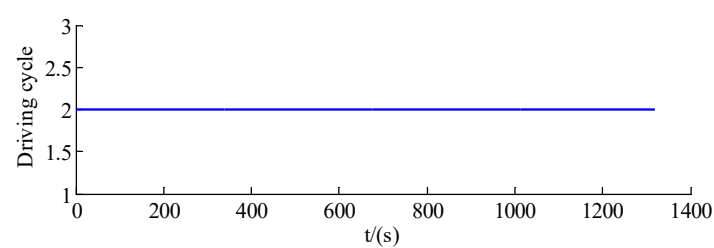

Fig. 14: Recognition result of CHINA

(1) In NEDC driving cycle, identity value of driving cycle is 2 before about $1,060 \mathrm{~s}$, and in about the interval [1060 , 1140] identity value of driving cycle is 3 , and converts back into 2 after about 1,140s. The result shows that the front of the low speed interval of NEDC is city driving cycle. The high speed interval of NEDC is high-speed driving cycle, in the high speed interval vehicle speed is more than $80 \mathrm{~km} / \mathrm{h}$, and the speed attaches to high speed according to the driving cycles recognition fuzzy logic rules. Whether the driving model places emphasis on economy or dynamic performance, driving cycle attaches high-speed driving cycle depending on fuzzy rule. Driving 
cycle in low-speed interval is recognized as city driving cycle. The simulation result conform to characteristic of NEDC.

(2) The identity value of CHINA is 2 in the whole simulation, that is, driving cycle is city driving cycle in the whole simulation. The simulation result conform to characteristic of CHINA. The results of NEDC and CHINA driving cycles show the validity of the driving cycles self-recognition model.

\subsection{Performance Simulation}

Fuel economy, exhaust emissions, and dynamic performance of vehicle only in CHINA driving cycle are conducted to verify the influence of driving cycles self-recognition strategy on vehicle performance. The curve of speed following performance, SOC of battery, and exhaust emissions in driving cycles self-recognition strategy (DCRS) and electric assistant control strategy (EACS) are shown as Fig. 15 and Fig. 16, while performance indexes are shown in Table 5.

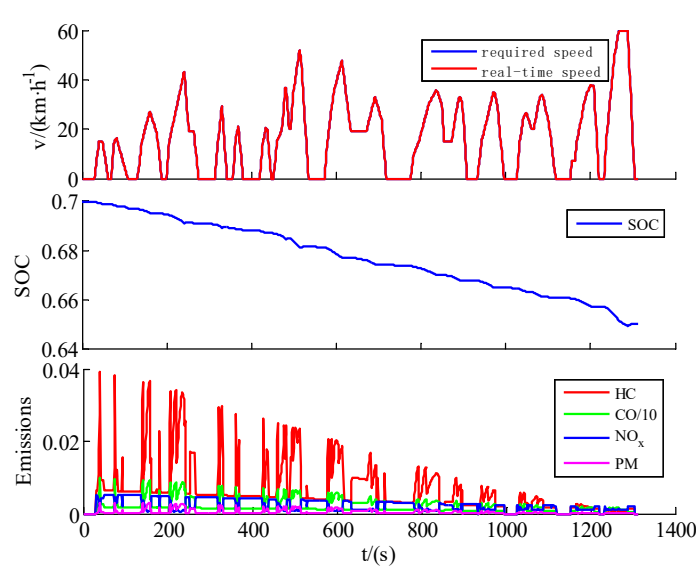

Fig. 15: Simulation results of DCRS

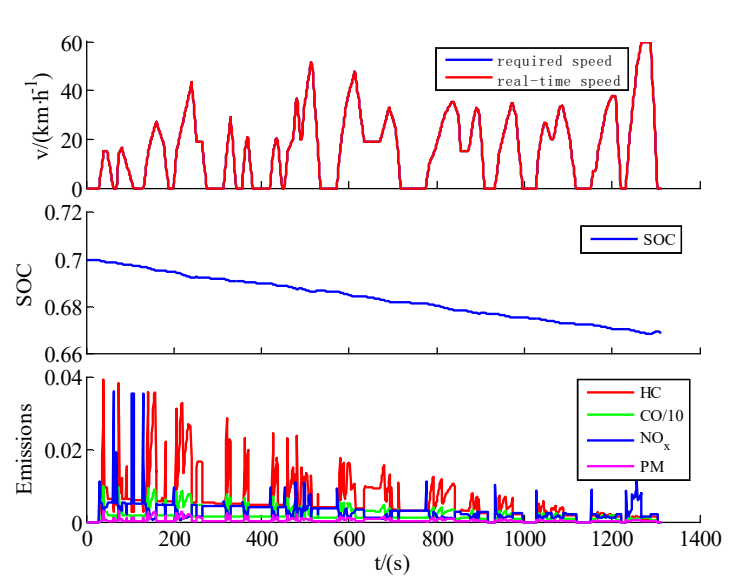

Fig. 16: Simulation results of EACS

Table 5: Performance results of CHINA driving cycles

\begin{tabular}{|c|c|c|c|}
\hline Performance & Index & DCRS & EACS \\
\hline Dynamic performance & $0-96.6 \mathrm{~km} / \mathrm{h}$ acceleration time $\mathrm{t}(\mathrm{s})$ & 12.4 & 9.9 \\
\hline Fuel economy & Fuel consumption $\mathrm{Q}(\mathrm{L} / 100 \mathrm{~km})$ & 5.4 & 6.2 \\
\hline \multirow{3}{*}{ Exhaust emissions } & $\mathrm{HC}(\mathrm{g} / \mathrm{km})$ & 1.471 & 1.3 \\
\cline { 2 - 4 } & $\mathrm{CO}(\mathrm{g} / \mathrm{km})$ & 3.552 & 4.164 \\
\cline { 2 - 4 } & $\mathrm{NOx}(\mathrm{g} / \mathrm{km})$ & 0.524 & 0.629 \\
\cline { 2 - 4 } & $\mathrm{PM}(\mathrm{g} / \mathrm{km})$ & 0.07 & 0.082 \\
\hline
\end{tabular}

The figures and the table above reflect that fuel consumption of the driving cycles self-recogniton strategy based on the multi-agent theory, when compared with the electric assistant control strategy reduces by $12.9 \%$, while $\mathrm{HC}$ increased by $13.2 \%$, CO reduced by $14.7 \%$, NOx reduced by $16.7 \%$, and $0-96.6 \mathrm{~km} / \mathrm{h}$ acceleration time increased by $25.3 \%$. The simulation results show that the fuel economy and exhaust emissions of driving cycles self-recognition strategy based on multi-agent theory is better than the electric assistant control strategy, but the dynamic performance is worse than the electric assistant control strategy. The acceleration time is less than 20s and still regarded as within the reasonable regions [10]. The SOC fluctuation range of DCRS is bigger than the EACS in the whole simulation, which indicates that the motor takes part in driving frequently, and this is one of the reason why fuel economy and exhaust emissions improves. DCRS can recognize CHINA driving cycle attaching to city driving cycles, and driving intention places emphasis on fuel economy, and exhaust emissions. So, fuel economy and exhaust emissions are improved but the dynamic performance is reduced. 


\subsection{CONCLUSION}

(1) Driving cycles self-recognition strategy based on the multi-agent theory can recognize current driving cycles depending on real-time vehicle and so on. that is, DCRS has a steady driving cycles recognition capability. It can match power of powertrain system according to the driving cycles, which is identified by the driving intention and average speed value.

(2) Driving cycles self-recognition strategy based on multi-agent theory can be adapted current driving cycles. Compared with conditional electric assistant control strategy, it can weigh fuel economy, exhaust emissions and dynamic performance of vehicle. Therefore, this research improves the vehicle's intelligent control level one step better then the existing solutions.

\section{ACKNOWLEDGMENT}

This paper was funded by the National Natural Science Foundation of China (Grant No. 51275002), Zhejiang Provincial Key Laboratory of Laser Processing Robot for the open fund (Grant No. lzsy-07), and Key Laboratory open fund for New Technology Application of Road Conveyance of Jiangsu Province (Grant No. BM20082061502). The authors acknowledge with thanks NSFC technical and financial support.

\section{REFERENCES}

[1] B. H. Wang, W. M. Wang, J. W. Zhang, et al, "Comparison Research of Different Control Strategies on Parallel Hybrid Electric Vehicle,” Journal of System simulation, Vol. 18, No. 2, 2006, pp. 401-404.

[2] Y. Zhang, H. P. Liu and Y.B, Wu. "Multi-objective control strategy for parallel HEV using fuzzy logic," Journal of Chongqing University, Vol. 36, No. 2, 2013, pp. 75-84.

[3] Z. Zhao and T. Wang, "Design and simulation of PHEV fuzzy logic control strategy," Beijing Automotive Engineering, No. 5, 2011, pp. 18-21.

[4] L. X. Zhao, S. G. Zuo and S. L. Lv, "Design of fuzzy logic control strategy of the parallel hybrid electric vehicle," Auto-motive technology, No. 1, 2007, pp. 24-27.

[5] C. E. Nino-Baron, A. R. Tariq, G. M. Zhu, et al, "Trajectory optimization for the engine-generator operation of a series hybrid electric vehicle," IEEE Transactions on Vehicular Technology, Vol. 60, No. 6, 2011, pp. 2438-2447.

[6] I. Kravtzoff, P. Dessante, J. C. Vannier, et al, "Global optimization of hybrid electrical system to decrease fuel consumption or operation cost," in 16th European Conference. on Power Electronics and Applications, Finland: Lappeenranta , 2014, pp. 479-385.

[7] Gh. Livinţ, V. Horga, M. Răţoi, et al, "Implementing the CANopen Protocol for the Distributed Control of a Hybrid Electric Vehicle," Electromotion 2009 - EPE Chapter 'Elec-tric Drives' Joint Symposium, France: Lille, 2009.

[8] A. Poursamad and M. Montazeri, "Design of genetic-fuzzy control strategy for parallel hybrid electric vehicles," Control Engineering Practice, Vol. 16, No. 7, 2008, pp. 861-873.

[9] Q. N, Wang, X. Z. Tang, P. Y. Wang, et al, "Control strategy of hybrid electric vehicle based on driving intention identification," Journal of Jilin University (Engineering and Technology Edition), Vol. 42, No. 4, 2012, pp. 789-795.

[10] T. C. Moore and A. B. Lovims, "Vehicle design strategies to meet and exceed PNGV goals," SAE paper No. 951906, USA: CA, 1995. 